\title{
Crecimiento y producción inicial de 15 especies de árboles tropica- les de la Amazonía ecuatoriana de estados sucesionales diferentes
}

\section{Growth and initial production of 15 tropical tree species from the Ecuadorian Amazonia with different successionel stages}

\author{
Herman Hernández Benalcázar1, Daniel Gagnon ${ }^{2}$, Robert Davidson ${ }^{3}$ \\ ${ }^{1}$ Universidad Central del Ecuador. Facultad de Ciencias Agrícolas. Carrera de Turismo Ecológico. Jerónimo Leiton y Av. La Gasca s/n. Ciudadela \\ Universitaria. 170521 Quito, Ecuador \\ ${ }^{2}$ University of Regina, Faculty of Science, Departement of Biology. Laboratory Building, LB244, 3737 Wascana Parkway, Regina, Saskatchewan, \\ S4SOA2, Canada \\ ${ }^{3}$ Institute of Environmental Sciences, Université du Québec à Montréal. Montreal, Canada
}

\begin{abstract}
Resumen
Se determinaron las características de crecimiento y de producción iniciales de 6 especies de árboles pioneros y de 9 especies de árboles de estados sucesionales avanzados, todas nativas de la Amazonia ecuatoriana. Las 15 especies fueron plantadas en un dispositivo experimental de bloques aleatorios completos, con 1 testigo y 2 tratamientos con fertilizantes. Los 2 tratamientos no difieren más que en el tiempo de liberación de los nutrientes (70 días y 180 días). El ensayo se situó en el recinto Domono, Macas, Morona Santiago, Ecuador. El crecimiento (altura, diámetro basal) de todas las plantas fueron medidas en 7 ocasiones durante los 6 meses que duró la experiencia. Tres recolecciones parciales de plántulas fueron efectuadas para medir la biomasa. Los resultados indican que Nectandra membranaceae es una de las especies de estados sucesionales avanzados que tienen mucha dificultad de adaptarse al crecimiento en plena luz, ya que su sobrevivencia fue casi nula. Las especies que tienen mejor crecimiento tanto en altura como en diámetro basal y biomasa son las especies pioneras: Heliocarpus americanus, Pollalesta discolor y Erythrina poeppigiana. El análisis de varianza sobre el efecto de los fertilizantes entre las especias, muestra que no hubo efectos significativos sobre el crecimiento en altura, diámetro basal y sobre la biomasa durante los tres primeros meses. Los resultados de este trabajo servirán para seleccionar de manera más acertada las especies pioneras susceptibles de producir lo más rápidamente una cubierta vegetal densa sobre sitios de suelos degradados y a las especies de árboles de estados sucesionales avanzados.
\end{abstract}

Palabras claves: árboles nativos, altura, diámetro, biomasa, suelo degradado, medio tropical húmedo.

\begin{abstract}
Growth and production initial characteristics of 6 pioner tree species and 9 advanced successional stages tree species were determined. The 15 species are native of the Ecuadorian Amazon. They were planted in a randomized complete blocks, with two fertilizer treatments (70 days and 180 days were the time of release of nutrients) and one witness. The study site is located in Domomo, Macas, Morona Santiago, Ecuador. Plant growth (height, basal diameter) was measured 7 times during 6 months. Three seedlings partial collections were made to measure the biomass. As result, Nectandra membranaceae is one of the advanced successional species with hard adaptation for growing in broad daylight, as its survival was almost zero. Heliocarpus americanus, Pollalesta discolor and Erythrina poeppigiana were the pionner species with better growth in height,
\end{abstract}


basal diameter and biomass. No significant effects were observed by fertilizer treatments on height growth, basal diameter and biomass during the first three months. The results will serve for selecting the pioneer species more susceptible to produce a dense vegetation cover on degraded soils and the tree species of advanced successional stages.

Keywords: native trees, height, diameter, biomass, degraded soils, humid tropical areas.

\section{Introducción}

Desde algunas décadas atrás, los bosques tropicales han atraído la atención tanto por la importancia en los procesos planetarios como por la impresionante diversidad de especies. De la misma manera que ha llamado la atención por la debilidad de producción de los suelos tropicales, después de poco tiempo de haber sido intervenidos (Fournier, 1978). La dinámica de este ecosistema tropical húmedo puede variar rápidamente, tomando diferentes direcciones según la perturbación y el grado de intervención. Las actividades humanas con frecuencia han causado deterioro acelerado de estos ecosistemas. Es verdad que actualmente se están practicando y ensayando algunos sistemas alternativos de manejo y control de los ecosistemas forestales, como por ejemplo: los sistemas silviculturales, agroforestales, agro-silvo-pastoriles, huertos caseros mixtos, los sistemas extractivistas, de rotación de cultivos, agricultura itinerante, etc (Peck 1990; Boese 1992; Lamprecht, 1990; Graaf \& Poels 1990; Bennett et al., 1994; Allegretti 1990).

Sin embargo, cada uno de ellos tienen sus ventajas y desventajas y algunos han sido cuestionados más que otros, Así por ejemplo, Peck (1990) manifiesta que las plantaciones de árboles de múltiples propósitos, pueden jugar un rol crítico en el establecimiento de la productividad, la estabilidad del ecosistema y la diversidad biológica de tierras degradadas de los trópicos. Pero, así mismo dentro de una perspectiva de desarrollo sostenido, Lamprecht (1990) plantea que en la selección de las especies arbóreas se requiere sumo cuidado. Tanto en la regeneración natural, como en las plantaciones ésta juega un papel preponderante. La regeneración natural garantiza la aptitud medioambiental. En general, esto es válido también para plantaciones con especies de árboles locales.

Las primeras etapas del establecimiento de plantaciones forestales son consideradas como unos sistemas biológicos jerárquicos (Margolis \&
Brand, 1990). A pesar de que son las condiciones medioambientales que controlan los procesos fisiológicos de las plantas y su ritmo de crecimiento (Margolis \& Brand, 1990), el hombre puede ayudar al restablecimiento de los ecosistemas forestales del mismo modo que los ha destruido, si logra comprender ciertos procesos ecofisiológicos, el comportamiento y la dinámica de las especies que componen una comunidad vegetal.

Una medida que puede ser de utilidad, es la correcta clasificación de las especies con las que no se tiene experiencia en uno de los tres grupos siguientes: en el de las pioneras, en el de las nómadas o en el de las especies esciófilas. En todo caso, la cuestión está en la optimización de todos los sistemas alternativos posibles de conservación y manejo de los bosques tropicales húmedos, a través de técnicas y procedimientos silvoculturales adecuados y ajustados a las condiciones medioambientales naturales que controlan los procesos fisiológicos y el ritmo de crecimiento.

Es necesario conocer las condiciones medioambientales óptimas requeridas por las especies para su regeneración y establecimiento (humedad, temperatura, nutrientes, materia orgánica del suelo, posibilidades de distribución, de germinación y competencia, etc.), la reacción de las plantas a nivel bioquímico, fisiológico o morfológico y las limitaciones en esos mismos parámetros.

Con este criterio, el propósito es encontrar especies de árboles pioneros que garanticen una rápida cubierta vegetal en suelos intervenidos y degradados de los trópicos húmedos. Esta condición permitirá el establecimiento de nuevas especies de sucesión secundaria avanzada en un sistema de regeneración natural del bosque. De igual manera la búsqueda de las especies de sucesión secundaria inicial y avanzada que cumplan múltiples propósitos y aporten de alguna manera al sostenimiento y subsistencia familiar a través de una utilidad inmediata y de largo plazo. 
Por último, es importante determinar los niveles de adaptabilidad y crecimiento de las especies nativas del trópico en condiciones adversas y favorables, para poder realizar la selección más acertada a fin de lograr mejores resultados en todos los sistemas alternativos de producción, manejo y conservación de los bosques tropicales húmedos y de esta manera contribuir al desarrollo sostenido de los trópicos.

Esta investigación apunta a determinar el crecimiento inicial en altura, diámetro basal y biomasa de 15 especies de árboles de la Amazonía ecuatoriana. Estas 15 especies de estados sucesionales diferentes fueron probadas sobre un suelo degradado con 2 tratamientos de fertilización y un testigo.

\section{Materiales y Métodos}

\subsection{Descripción del sitio experimental}

El sitio experimental está situado en una hacienda ubicada aproximadamente a $10 \mathrm{~km}$ al norte de la ciudad de Macas, en el sector Domono, provincia de Morona Santiago, Ecuador. Geográficamente se encuentra entre $02^{\circ} 06^{\prime}$ Lat. S. y $78^{\circ} 06^{\prime}$ Long. W. a una altitud de 1,300 m.s.n.m. La zona se caracteriza por la presencia de colinas bajas y planicies entrecortadas por pequeños ríos afluentes del río Upano que es la principal cuenca hidrográfica de la región.

La temperatura media anual es de $22^{\circ} \mathrm{C}$ y la precipitación total anual de 3,000 mm. De acuerdo a Cañadas (1983), esta área corresponde a la región bioclimática húmeda subtropical y zona de vegetación de bosque muy húmedo Pre-Montano (bmhPM). En cuanto a la composición y estructura de las formaciones vegetales tropicales, corresponde a la de los bosques húmedos siempre verdes deciduos (Lamprecht, 1990).

La plantación se realizó en un terreno abandonado después de haber mantenido un cultivo de caña de azúcar y se muestra que es un suelo altamente degradado. Hay predominio de plantas herbáceas no útiles como alimento de ganado.

\subsection{Crecimiento de las especies de árboles en experimentación}

Se observó el crecimiento inicial de 15 especies de árboles tropicales de la Amazonía ecuatoriana durante 6 meses. Seis especies de sucesión secundaria inicial y 9 especies de sucesión secundaria avanzada. Las especies de sucesión secundaria inicial son: Heliocarpus americanus Linnaeus, Pollalesta discolor Kunt, Erythrina poeppigiana. Inga densiflora Bentham, Piptadenia pteroclada Bentham y Croton Lechleri Muell. Las especies de sucesión secundaria avanzada son: Cordia alliodora (Ruiz \& Pavón) Oken, Cedrela odorata Linneaus, Cariodendron orinocense Karsten, Virola dukei A.C.Smith, Ocotea floccifera, Platymiscium pinnatum, C. DeCandolle, Clarisia biflora Ruiz \& Pavón, Nectandra sp. y Nectandra membranacea (Swart) Grisebach.

Para la selección de las especies experimentales se consideraron las siguientes condiciones: la presencia de árboles semilleros en el lugar de plantación o en lugares cercanos, utilidades que pueden prestar cada una de las especies, la posibilidad de encontrar semillas para la siembra y la reproducción de plántulas, el tiempo y el índice de germinación.

Las 6 especies pioneras seleccionadas son de rápido crecimiento, capaces de producir una cubierta vegetal en corto tiempo. P. discolor, H. americanus y $C$. lechleri, tienen gran facilidad de dispersión. A estas especies se las encuentra entre las primeras colonizadoras en terrenos removidos, en las orillas de las carreteras y en terrenos degradados por uso excesivo. A I.densiflora se la encuentra generalmente en cultivos, formando parte de los árboles frutales. Erythrina poeppigiana, es una especie que se la está usando para la construcción de cercas vivas y se la siembra en los pastizales para la producción de sombra y fijación de nitrógeno. P. pteroclada es una Mimoseaseae que crece en los pastizales y los campesinos la mantienen porque produce madera de buena calidad. C. lechleri tiene propiedades medicinales, su latex es utilizado como cicatrizante de heridas y ulceraciones y tiene gran valor comercial. $P$. discolor es una especie muy comercial, su madera se la utiliza para la construcción de cajas de embalaje para el transporte de frutas, especialmente de naranjila (Solanum quitoensis Lam).

Las especies de sucesión secundaria avanzada, son de crecimiento lento pero pueden alcanzar dimensiones mayores a los $25 \mathrm{~m}$ de altura y $1 \mathrm{~m}$ de diámetro del fuste (ADP). Son tolerantes a la sombra y necesitan de la protección de las especies pioneras inicialmente. La utilidad, generalmente es la producción de madera de buena calidad. 
Algunas especies de sucesión secundaria avanzadas son muy conocidas por su amplia distribución ecológica y por la calidad de madera que proporcionan. Cordia alliodora, Cedrela odorata y Platymiscium pinnatum, por ejemplo, son especies domesticadas y adaptadas a condiciones exigidas por especies pioneras. Nectandra sp. y Ocotea floccifera son especies muy explotadas por su calidad de madera. A Nectandra sp. se la encuentra en el interior de los bosques naturales y también en campos abiertos. O. floccifera se la encuentra unicamente en el interior del bosque. Virola dukei produce madera de buena calidad, se la usa para la construcción de viviendas y los indígenas de la Amazonia le dan otros usos por su contenido de alcaloides (Bennett \& Alarcón, 1993). Cariodendron orinocense es una especie que produce madera de regular calidad, pero las semillas son cosechadas por los indígenas para su alimentación porque contienen un alto porcentaje de aceites y proteínas. A esta especie se la encuentra tanto en los bosques naturales como en campos abiertos. Finalmente, Clarisia biflora produce madera de buena calidad y también de estos árboles se extrae el látex que tiene propiedades medicinales.

\subsection{Recolección de las semillas}

Las plántulas se reprodujeron en un vivero a partir de semillas recolectadas durante el mes de junio, a excepción de Platymiscium pinnatum, Erythrina sp, Ocotea floccifera y Nectandra sp. por haber pasado la época de cosecha. Sin embargo, las plántulas se las encontró en el interior del bosque natural.

\subsection{Dispositivo experimental}

En una superficie de $972 \mathrm{~m}^{2}$ de terreno se establecieron 3 bloques y cada uno de ellos divididos en 45 parcelas de $6 \mathrm{~m}^{2}$ cada una, dando un total de 135 parcelas (15 especies, 3 tratamientos, 3 bloques). La selección de las parcelas para cada especie y tratamientos se realizó de manera aleatoria.

En cada parcela se sembraron 15 plántulas de la misma especie. Las plantas fueron numeradas del 1 al 15 con la finalidad de facilitar el trabajo de medición y registro. En todo el dispositivo se plantaron 2,025 plantas de 15 especies. Se aplicaron tres tratamientos, incluido el testigo. Los dos tratamientos consisten en adicionar $20 \mathrm{~g}$ de fertilizantes que contienen los mismos elementos químicos: N, P y K y la misma estructura en su composición química, pero que difieren solo en el tiempo de liberación de los nutrientes (70 y 180 días). Se hicieron 7 mediciones de altura y diámetro basal: una por cada mes durante los 6 meses de experimentación, más una medida inicial. Estas dos medidas se tomaron de manera simultánea. La biomasa se midió en tres ocasiones; una inicial, otra a los 3 meses y la última a los 6 meses de crecimiento. Solo se calculó la biomasa de la parte aérea de las plantas, es decir, hojas y tallos utilizando el método de pesajes en el laboratorio de la Universidad del Azuay.

Para el análisis del suelo, se seleccionaron al azar 9 parcelas por cada bloque, es decir, 27 parcelas en total. En estas parcelas seleccionadas se midió la temperatura y se tomaron las muestras de suelo (27 muestras) para el cálculo de la humedad, el pH, la materia orgánica y la determinación de los bio-elementos. Las muestras de suelo se tomaron en una profundidad de entre 5 y $10 \mathrm{~cm}$. La temperatura se midió una vez cada mes, en una profundidad de 10 $\mathrm{cm}$. La humedad se calculó una vez cada mes y se utilizó el método de pesajes. Este análisis se realizó en la Universidad del Azuay.

El cálculo de la materia orgánica del suelo se realizó en los laboratorios de la Universidad de Quebec en Montreal (UQAM) por dos ocasiones, utilizando el método de calcinación y pesajes. Para la extracción del $\mathrm{NO}_{3}$ y del $\mathrm{NH}_{4}$ se utilizaron $10 \mathrm{~g}$ de suelo seco desmenuzado (partículas $<100$ mesh) disueltos en $40 \mathrm{ml}$ de $\mathrm{KCl} 2 \mathrm{M}$ como extractante. El aparato de detección fue el Tecator Fiastar. Para la extracción del fósforo asimilable se utilizó el método Bray. Para la detección se usó el Espectro-fotómetro. Estos dos análisis se los realizó en los laboratorios del Biodome de Montreal.

La extracción de los cationes $\mathrm{K}^{+}, \mathrm{Ca}^{+}, \mathrm{Mg}^{+}$ y $\mathrm{Mn}^{+}$se hizo utilizando el método sugerido por Stewart (1989). La extracción del Fe y Al, se hizo mediante el método descrito por Hendershot y Lalande (1993), utilizando el espectro de absorción atómica de la UQAM. De acuerdo al plan experimental, se utilizó la ANOVA, para la comparación múltiple de las medias y la prueba de significación de Tukey como modelos de análisis estadístico. Se hizo el análisis de los diferentes índices de crecimiento de las planta tanto en altura como en diámetro basal y biomasa total (Hunt, 1990): tasa de crecimiento relativo (RGR) en altura por cada mes, RGR en diámetro basal y RGR en biomasa. 


\section{Resultados y Discusión}

\subsection{Suelo}

El análisis de varianza detecta un efecto bloque para la materia orgánica y $\mathrm{NO}_{3}$ del suelo, tanto en el tiempo inicial como en el tiempo final del experimento. Para las variables $\mathrm{Mg}, \mathrm{Al}, \mathrm{P}_{\mathrm{N} \mathrm{NH}}$ el efecto bloque se detecta solamente en el tiempo inicial, en cambio para el $\mathrm{Ca}, \mathrm{Mn}$ el efecto bloque se detecta en el tiempo final. En cuanto a los tratamientos se observa que hay efecto de estos solamente en la variable $\mathrm{P}$ en el tiempo final (Tabla 1). A pesar de que la concentración de $\mathrm{P}$ es muy baja, se pudo detectar una diferencia estadísticamente significativa entre los tratamientos, encontrándose mayor contenido de $\mathrm{P}$ en el tratamiento 2 con relación al testigo y al tratamiento 3 .

Se deduce que el suelo y la luz son los recursos más importantes que habrían influido en el crecimiento de las 15 especies, cuando ANOVA detecta un efecto bloque al inicio y al final del experimento, especialmente para la materia orgánica y el $\mathrm{NO}_{3}$. Por otra parte se pudo observar que en las especies de menor crecimiento se produce foto inhibición por la presencia del color amarillo de sus hojas, provocando a su vez la gran variación de crecimiento individual entre unos y otros árboles de la misma especie.

De igual manera, observadas las características del suelo donde se realizó el experimento (Tabla 1) se puede detectar que hay una deficiencia absoluta de fosforo asimilable y bajos niveles de los demás elementos nutritivos. La deficiencia de nutrientes más las variaciones de humedad, temperatura y compactación, seguramente habría afectado directamente al desarrollo de las raíces de las plantas individualmente.

Las medias de crecimiento anual en altura revelan que hay una tendencia positiva del efecto de los tratamientos en el crecimiento. Se puede afirmar que la mayor tasa de crecimiento tanto en altura como en diámetro basal y biomasa la tienen las especies Pollalesta discolor, Heliocarpus americanus y Erythrina sp seguidas por Cordia alliodora y Cedrela odorata.

Tabla 1. Características del suelo del dispositivo experimental.

\begin{tabular}{lll}
\hline & $\begin{array}{l}\text { Noviembre 1993 } \\
\mathrm{n}=27\end{array}$ & $\begin{array}{l}\text { Mayo 1994 } \\
\mathrm{n}=27\end{array}$ \\
\hline $\mathrm{pH}$ & 5.53 & 5.27 \\
\hline $\mathrm{NO}_{3}(\mathrm{ppm})$ & 13 & 37 \\
$\mathrm{NH}_{4}(\mathrm{ppm})$ & 111 & 92 \\
$\mathrm{P}(\mathrm{ppm})$ & $\mathrm{O}^{*}$ & $\mathrm{O}^{*}$ \\
$\mathrm{~K}(\mathrm{ppm})$ & 155 & 186 \\
$\mathrm{Ca}(\mathrm{ppm})$ & 174 & 191 \\
$\mathrm{Mg}(\mathrm{ppm})$ & 59 & 81 \\
$\mathrm{Al}(\mathrm{ppm})$ & 43 & 54 \\
$\mathrm{Mn}(\mathrm{ppm})$ & 9 & 5 \\
\hline $\mathrm{Materia} \mathrm{orgánica} \mathrm{( \% )}$ & 10.7 & 10.8 \\
\hline
\end{tabular}

\subsection{Sobrevivencia y crecimiento de los árboles}

Las ANOVAs de altura, diámetro basal y biomasa total, demuestra que siempre hay una dife- rencia significativa de crecimiento entre las especies estudiadas (Tabla 2). 
Tabla 2. Sumario de análisis de varianza de RGR de variables de crecimiento.

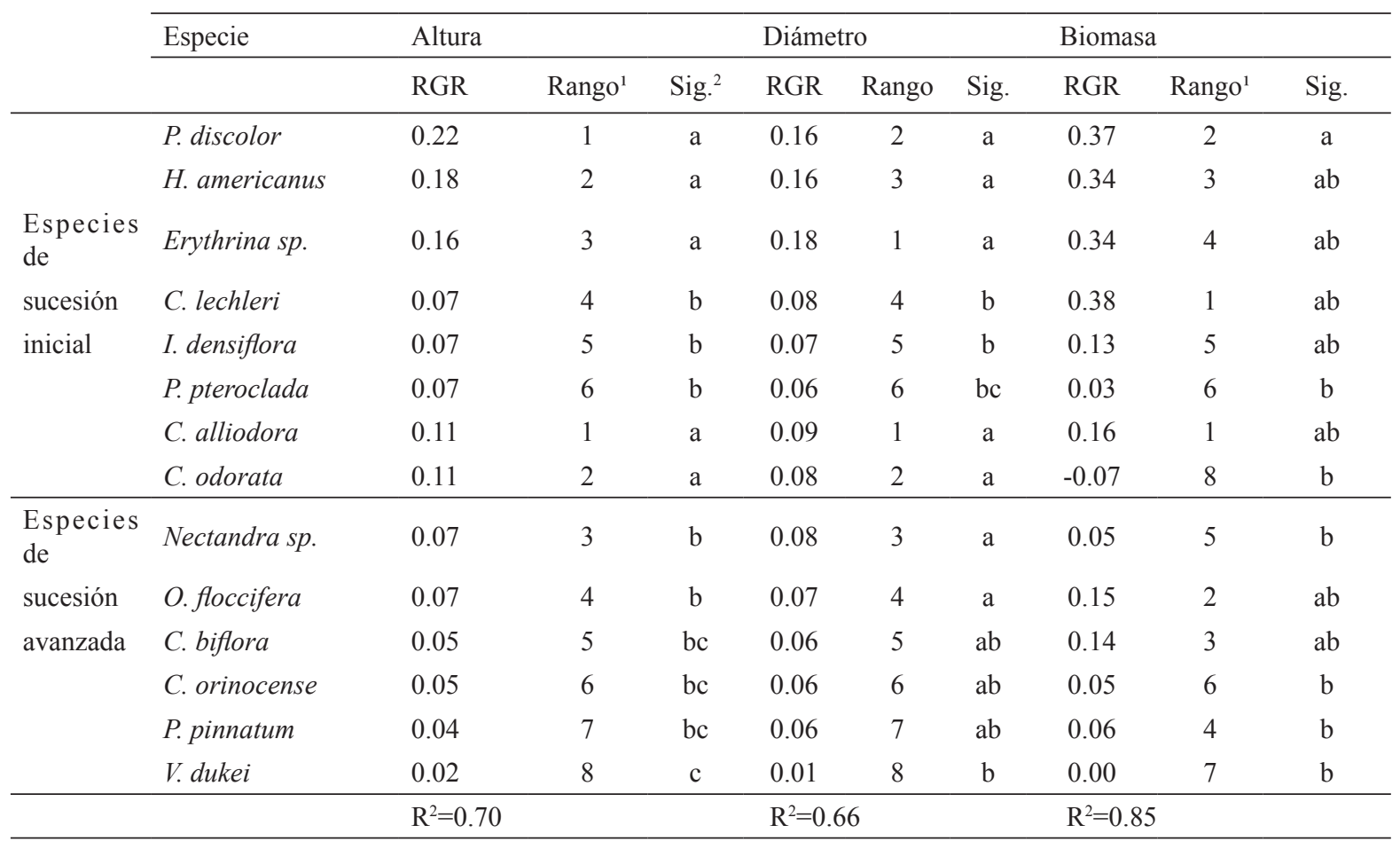

1 Rango separado por especie inicial y especies de sucesión inicial y especies de sucesión avanzada.

2 Significación de diferencias estadísticas para todas las especies; la misma letra significa no diferencia estadística.

De acuerdo a lo que se observa en el Cuadro 1, sobre el RGR (Relative Growth Rate), se identifican tres grupos definidos. En el primer grupo se ubican: Pollalesta discolor, Erythrina sp y Heliocarpus americanus, a las cuales les identificaríamos como heliófilas efímeras, porque su ciclo de vida lo cumplen solamente en sitios abiertos y tienen una vida relativamente corta (10 - 15 años). Los sistemas de fotosíntesis de estas especies son muy eficaces en plena luz y como consecuencia de esta estrategia su madera es liviana, suave y de poca fuerza y resistencia, pero con una gran capacidad para producir materia orgánica que cubre rápidamente el suelo. El segundo grupo lo constituyen: Cordia alliodora, Cedrela odorata, Piptadenia pteroclada e Inga densiflora, a las cuales las consideraríamos como heliófilas durables porque mantienen un conjunto de características que les permite la explotación de sitios abiertos y los ocupan durante períodos más largos que las heliófilas efímeras, la capacidad de fotosíntesis es intermedia y el crecimiento es más o menos rápido, la calidad de madera es buena por ser moderadamente densa y moderadamente liviana. Este grupo de especies es sumamente interesante para la producción forestal tanto en bosques naturales como en plantaciones y sistemas agroforestales. En el tercer grupo se ubicarían: Platimiscium pinnatum, Cariodendron orinocense, Clarisia biflora, nectandra sp, Ocotea floccifera y Virola duckey, a las mismas que las consideraríamos como esciófitas parciales en razón de que el patrón general de asignación de recursos es de un crecimiento más lento y de inversión en la producción de estructuras permanentes más duraderas que las heliófitas (Finegan, 1992). Por último a Nectandra membranacea, por no haber sobrevivido a la plantación a plena luz la ubicaríamos en el grupo de las esciófitas totales.

A nivel de sobrevivencia la mayoría de especies muestran una tasa mayor del $95 \%$. Solamente Nectandra membranacea muestra una tasa de sobrevivencia inferior al 5\%, en cambio Nectandra sp mantiene una sobrevivencia aproximada del 60\%. Estos resultados son atribuibles posiblemente a una mala aclimatación de las plántulas silvestres.

\section{Conclusiones}

Los resultados del análisis del suelo donde se realizó la plantación experimental evidencian una acentuada degradación al inicio de la plantación y después 
de 6 meses se nota una pequeña recuperación de $\mathrm{K}, \mathrm{Ca}$ y $\mathrm{Mg}$, sin embargo, en comparación de otros suelos degradados el $\mathrm{P}$ asimilable se mantiene en 0 (ppm) el Al se incrementa, aumenta levemente la acidez y no se observa incorporación de materia orgánica.

Más del 95\% de las plantas de las especies estudiadas sobrevivieron en condiciones adversas de suelos degradados, a excepción de las plantas de Nectandra membranacea.

Se identificaron tres grupos de especies más o menos definidos que aprovechan de diferentes maneras la asignación de recursos: el grupo de las heliofitas efímeras, en el que se ubican las pioneras Pollalesta discolor, Erythrina poepigiana y Heliocarpus americanus; el grupo de las heliófitas durables en el que se ubican: Cedrela odorata, Cordia alliodora, Piptadenia pteroclada e Inga densiflora. El tercer grupo es el de las esciófitas parciales en el que se ubican: Platymiscium pinnatum, Cariodendron orinocense, Clarisia biflora, Nectandra sp, Ocotea floccifera y Virola duckei.

Los tratamientos con abonos no influyeron significativamente en el crecimiento de altura, diámetro basal y biomasa de las plantas, con relación al testigo.

\section{Referencias}

Allegretti, M. (1990). Extractive Reserves: An alternative for reconciling development and environmental conservation in amazonia. Altrnatives to Deforestation Steps Toward Sustainable Use of the Amazon Rain Forest. New York: Columbia University Press. 252-264.

Bennett, B., \& Alarcón, R. (1994). Osteophloeum Platyspermum and Virola dukei (Miristicaceae): New ly reported as hallucinogens from amazonian Ecuador. Económic Botany, 48(2), 152 - 158.

Boese, E., (1992). Actividades forestales y silviculturales en la región Amazónica ecuatoriana: Experiencias y resultados 1985 - 1990, Lumbaqui, provincia de Sucumbíos.

Cañadas, L. (1983). El mapa bioclimático y ecológico del Ecuador, Ministerio de Agricultura y Ganadería del Ecuador. Quito, Ecuador: PRONAREG.

Fournier, F., (1978). Balance hídrico, Ecosistemas de los bosques tropicales, Informe UNESCO/PNUMA/FAO.
Las 14 de las 15 especies de árboles tropicales estudiadas en la Amazonía ecuatoriana son aptas para iniciar plantaciones forestales mixtas en suelos degradados y sin la utilización de abonos.

El efecto de los tratamientos se detecta solamente a partir del cuarto mes de crecimiento. El tratamiento 2 presenta un efecto estadísticamente significativo en el crecimiento de Clarisia biflora y el tratamiento 3 para la especie Cedrela odorata entre la especies de sucesión secundaria avanzada. Así mismo, entre las especies pioneras se observa que el tratamiento 3 ejerce mayor efecto en el crecimiento de Piptadenia pteroclada a partir del cuarto mes.

En el mes de marzo se produce un crecimiento acelerado en la mayoría de las especies, lo que se demuestra que el aumento de la precipitación a ciertos niveles, puede detener el crecimiento de las especies estudiadas.

Las especies que más biomasa producen son: $P o-$ llalesta discolor, Heliocarpus americanis y Herythrina poepigiana, entre las plantas pioneras y entre las de sucesión avanzada se destacan: Caryodendron orinocense, Cordia alliodora y Cedrela odorata.

Graaf, N., \& Poels, R. (1990). The CELOS, Management System: A polycyclic method for sustained timber productin in south american rain forest. Altrnatives to Deforestation Steps Toward Sustainable Use of the Amazon Rain Forest. New York: Columbia University Press.

Hunt, R. (1990). Basic growth analysis. Plants growth analysis for baguinnes. London Un Win Hayman.

Lamprecht, H. (1990). Silvicultura en los Trópicos. Los ecosistemas forestales en los bosques tropicales y sus especies arbóreas. Posibilidades y métodos para un aprovechamiento sostenido. Instituto de Silvicultura de la Universidad Göttigen. Cooperación técnica - República Federal de Alemania.

Peck, R. (1990). Programing agroforestry practices among small producers: The case of the Coca agroforestry proyect in amazonian Ecuador. Cap. II. Alternatives to Deforestation Steps Toward Sustainable Use of the Amazon Rain Forest. New YorK: Columbia University Press. 